\title{
Video Article \\ Intact Histological Characterization of Brain-implanted Microdevices and Surrounding Tissue
}

\author{
Andrew J. Woolley ${ }^{1}$, Himanshi A. Desai ${ }^{1}$, Janak Gaire ${ }^{2}$, Andrew L. Ready ${ }^{1}$, Kevin J. Otto ${ }^{1,2}$ \\ ${ }^{1}$ Weldon School of Biomedical Engineering, Purdue University \\ ${ }^{2}$ Department of Biological Sciences, Purdue University
}

Correspondence to: Andrew J. Woolley at awoolley@purdue.edu

URL: https://www.jove.com/video/50126

DOI: doi: $10.3791 / 50126$

Keywords: Neurobiology, Issue 72, Neuroscience, Biomedical Engineering, Medicine, Central Nervous System, Brain, Neuroglia, Neurons, Immunohistochemistry (IHC), Histocytological Preparation Techniques, Microscopy, Confocal, nondestructive testing, bioengineering (man-machine systems), bionics, histology, brain implants, microelectrode arrays, immunohistochemistry, neuroprosthetics, brain machine interface, microscopy, thick tissue, optical clearing, animal model

Date Published: 2/11/2013

Citation: Woolley, A.J., Desai, H.A., Gaire, J., Ready, A.L., Otto, K.J. Intact Histological Characterization of Brain-implanted Microdevices and Surrounding Tissue. J. Vis. Exp. (72), e50126, doi:10.3791/50126 (2013).

\section{Abstract}

Research into the design and utilization of brain-implanted microdevices, such as microelectrode arrays, aims to produce clinically relevant devices that interface chronically with surrounding brain tissue. Tissue surrounding these implants is thought to react to the presence of the devices over time, which includes the formation of an insulating "glial scar" around the devices. However, histological analysis of these tissue changes is typically performed after explanting the device, in a process that can disrupt the morphology of the tissue of interest.

Here we demonstrate a protocol in which cortical-implanted devices are collected intact in surrounding rodent brain tissue. We describe how, once perfused with fixative, brains are removed and sliced in such a way as to avoid explanting devices. We outline fluorescent antibody labeling and optical clearing methods useful for producing an informative, yet thick tissue section. Finally, we demonstrate the mounting and imaging of these tissue sections in order to investigate the biological interface around brain-implanted devices.

\section{Video Link}

The video component of this article can be found at https://www.jove.com/video/50126/

\section{Introduction}

The field of neuroprosthetic research aims to assist individuals suffering from various disabilities and disorders by bypassing diseased or damaged structures in the body through CNS interfacing devices ${ }^{1,2}$. Brain-implanted microdevices, such as microelectrode arrays (MEAs), can be used to record or stimulate brain structures, and thus allow the establishment of long-term interfaces between electronics and CNS tissue e $^{3-5}$ Penetrating MEAs, devices which are driven into the brain tissue, hold particular promise as bi-directional interfaces due to the close proximity within which they present electrodes to a relatively small set of nearby neurons ${ }^{6}$.

However, complex tissue responses result from the long-term implantation of penetrating MEAs, often resulting in variable and gradually degrading electrophysiological signal-to-noise ratios over days to months, and an increase in electrical impedance between electrode sites and ground ${ }^{7,8}$. The putative origins of these changes include the activation of microglia, reactive astrocytosis along the microdevices, and a loss or migration of neurons from the tissue surrounding to implanted devices ${ }^{9-11}$. A major challenge to understanding these tissue changes around chronic, penetration MEAs is the difficulty in capturing histological data of the intact tissue interface surrounding chronically implanted devices ${ }^{12}$ Histological analysis of the tissue with the device/tissue interface still present would improve upon the current device-removal histological protocols. With an undisturbed device remaining in the tissue, the biological impact of relatively subtle interactions, such as the utilization of biocompatible coatings ${ }^{13,14}$ or the electrical clearing of the electrode surface ${ }^{15,16}$, could be imaged and analyzed with respect to the implant.

Here we demonstrate a method to collect, process, and image the intact microdevice interface for detailed microscopy-based analysis of the surrounding brain tissue. In this method, the device and surrounding brain tissue are collected within a thick (>250 $\mu \mathrm{m})$ tissue section using a vibratome. To improve histological label penetration into these thick slices, fluorescent histochemical and immunohistochemical labels are applied at high concentrations in solutions containing blocking serum and detergent for multiple days. An optical clearing solution is employed to improve microscopy imaging depths, and the tissue is mounted in 2-sided chambers for subsequent laser scanning confocal microscopy ${ }^{17}$. To capture the full histological interface, a computer-controlled translational stage is used during imaging to collect z-stack panoramas along the length of implants. In addition to imaging applied tissue labels, collecting laser reflectance back from implants and transmission light through the tissue both help localize the device interface in relation to surrounding tissue. Tissue prepared using this "Device-Capture Histology" (DCHist) protocol provides imaging access to the morphologically preserved tissue/device interactions, and thus improves upon previous device-removal histological protocols ${ }^{18}$. 


\section{Protocol}

\section{Solutions}

Phosphate Buffered Saline (PBS) - in g/l; $9 \mathrm{~g} \mathrm{NaCl}, 0.144 \mathrm{~g} \mathrm{KH}_{2} \mathrm{PO}_{4}, 0.795 \mathrm{~g} \mathrm{Na}_{2} \mathrm{HPO}_{4}$, at pH 7.4

4\% Formaldehyde - in $\mathrm{ml} / \mathrm{l} ; 202 \mathrm{ml}$ sodium phosphate dibasic solution $\left(0.4 \mathrm{M} \mathrm{Na}_{2} \mathrm{HPO}_{4}\right), 48 \mathrm{ml}$ sodium phosphate monobasic solution (0.4 M $\mathrm{NaH}_{2} \mathrm{PO}_{4}$ ), $500 \mathrm{ml} 8 \%$ formaldehyde solution, $250 \mathrm{ml}$ Milli-Q DDi water, at $\mathrm{pH} 7.4$

HEPES Buffered Hank's Saline with Sodium Azide (HBHS) - in g/l; $7.5 \mathrm{~g} \mathrm{NaCl}, 0.3 \mathrm{~g} \mathrm{KCl}, 0.06 \mathrm{~g} \mathrm{KH}_{2} \mathrm{PO}_{4}, 0.13 \mathrm{~g} \mathrm{Na}_{2} \mathrm{HPO}_{4}, 2 \mathrm{~g} \mathrm{glucose} 2.4 \mathrm{~g}$ HEPES, $0.05 \mathrm{~g} \mathrm{MgCl}_{2} 6$ parts $\mathrm{H}_{2} \mathrm{O}, 0.05 \mathrm{~g} \mathrm{MgSO}_{4} 7$ parts $\mathrm{H}_{2} \mathrm{O}, 0.165 \mathrm{~g} \mathrm{CaCl}_{2}, 0.09 \mathrm{~g} \mathrm{NaN}_{3}$ at pH 7.4

Wash Solution (WS) - $1 \%$ Vol/Vol, Normal Goat Serum, $0.3 \%$ Triton X-100, in HBHS with sodium azide $\left(\mathrm{NaN}_{3}\right)$. Refrigerate the WS at $4{ }^{\circ} \mathrm{C}$ before use in subsequent steps.

U2 Scale Solution - $4 \mathrm{M}$ urea, $30 \%$ glycerol and $0.1 \%$ Triton $\mathrm{X}-100{ }^{17}$

\section{PROCEDURE}

All experiments were done under the supervision of the Purdue Animal Care and Use Committee and the Laboratory Animal Program at Purdue University.

\section{Surgery}

1. Various aseptic surgical methods are compatible with the presented histological method. The use of a stereotaxic frame and automated inserters are recommended to improve reproducibility and control of the microelectrode arrays (MEA) implantation angle with respect to stereotaxic planes.

Note: In this demonstration our surgical method is as follows: hold the rodent subject in stereotaxic earbars while under isoflurane anesthetic (between 1-3\%) carried by medical grade oxygen. Test for the lack of a toe-pinch reflect in the rat or lack of a tail pinch reflex in the mouse to confirm that the animal is fully anesthetized. Apply eye ointment and clean the surgical site with three alternating washes of Betadine and ethanol. Wash hands, dawn surgical gloves, hairnet, facemask and gown. Inject a bolus of Lidocaine to numb the surgical area, create an incision using scissors or a scalpel, clear periosteum with bone scraper and cotton applicators, and create a craniotomy using a dental drill handpiece and drill bit. Drive a single-shank MEA into the cortex using a micromanipulator. Have a surgical assistant aid in maintaining aseptic surgery conditions and document the progress of the surgery.

2. Following implantation of the MEA into the brain, collect images through a surgical microscope to inform the eventual removal of the skull from around the brain. Implanted portions of the devices will be preserved in situ.

Note: The eventual collection of tissue with in situ devices relies on closely matching the plane of device insertion with the plane of tissue sectioning. Detailed notes about the device insertion plane relative to the brain are thus very important.

3. After device implantation, apply the silicone elastomer Kwik-Sil (WPI), around any exposed MEA shanks or cabling, and allow to cure. A sterilized plastic piece, such as a cut pipette tip, may be used to help contain the silicone elastomer in a small well around the craniotomy while curing.

4. Apply a layer of two-part dental acrylic ("Jet Liquid" and "Jet Powder" from Lang Dental) over the Kwik-Sil and any exposed skull.

Note: In a later step, the headcap will be melted through to allow for the implant to be separated from the skull. UV-curing acrylic should be avoided over the Kwik-Sil and craniotomy, as this very hard acrylic is difficult to remove later. Visually opaque materials around the implant should also be avoided; clear Kwik-Sil provides a view of the implant during subsequent steps of this protocol.

5. Perform post-operative care procedures as per the local protocol of the animal welfare regulations, and return ambulatory subjects to singlehoused cage-boxes.

\section{Perfusion and Tissue Collection}

Note: See Gage et al. for a detailed perfusion procedure walkthrough in the rat animal model ${ }^{19}$.

1. Use the anesthesia and perfusion protocol approved by the institution's animal care and use committee. After deeply anesthetizing the rodent (confirmed by a lack of toe-pinch reflex in rat or tail-pinch reflex in mice) and exposing the heart, make shallow scissor cuts into the left ventricle and right atrium. Insert a blunt needle into the left ventricle and deliver room temp PBS. Deliver a total of $\sim 10 \mathrm{ml} \mathrm{PBS}$ at $\sim 5 \mathrm{ml} / \mathrm{min}$ in mice and $\sim 200 \mathrm{ml}$ PBS at $\sim 100 \mathrm{ml} / \mathrm{min}$ in rats. Look for blood clearance from the liver during.

2. Inject histologically relevant chemical labels transcardially, if so desired, by syringe delivery with care taken to avoid introducing bubbles.

Note: Vascular labels (e.g. Dil) and nucleic acid counterstain dyes (e.g. Hoechst 33342) are examples of chemical labels deliverable during perfusion. When administered properly, these histological markers should label the whole animal ${ }^{20}$. 
3. Deliver buffered, room temperature, $4 \%$ formaldehyde transcardially to fix the animal. Avoid septum perforation across the heart, which can be evidenced by lung-nose drainage during perfusion. Look for fixation tremors in the large muscles to indicate complete perfusion. Deliver a total of $\sim 10 \mathrm{ml}$ fixative at $\sim 5 \mathrm{ml} / \mathrm{min}$ in mice and $\sim 200 \mathrm{ml}$ fixative at $\sim 100 \mathrm{ml} / \mathrm{min}$ in rats.

4. Following perfusion, decapitate the animal, expose part of the brain stem or cerebellum using rongeurs or scissors, and place head in $4 \%$ formaldehyde solution for overnight at $4{ }^{\circ} \mathrm{C}$.

5. Wash the head in three changes of PBS with one to four hour intervals between washes

6. Store fixed heads in HBHS containing sodium azide at $4{ }^{\circ} \mathrm{C}$ for days to months.

\section{Brain Removal with in situ Devices}

Note: Perform this section in a fume hood while wearing appropriate personal protective equipment. Avoid tissue desiccation by returning the fixed head to HBHS solution periodically.

1. Carefully dissect away skin and other tissues from around the acrylic skull cap using forceps and small scissors.

2. While wearing heat-insensitive gloves, use a soldering iron to remove dental acrylic and expose an area of underlying clear Kwik-Sil (Figure 2a).

Note: The goal of this step is to allow micro-scissors an appropriate angle of access to positions where the implanted devices enter the brain, so that brain-implanted components may be separated from components secured to the skull.

3. Under a surgical microscope, cut into the silicone elastomer with curved micro-scissors, removing small pieces of Kwik-Sil with tweezers down to the position where the devices enter the brain.

4. Continue cutting along the surface of the craniotomy with curved micro-scissors to separate device components attached to polysilicon and skull from components implanted in the brain. Again use microscope magnification and great care when cutting through the exposed device components, taking care to avoid pushing or dragging the implants in the fixed tissue.

5. Remove bone around the headcap with care using rongeurs. Use a spatula to separate the brain with implanted devices from the skull. Store the brain in HBHS solution until ready to slice.

6. Place the brain in a glass Petri dish filled with HBHS, and use a razor blade to remove extraneous tissue, such as spinal cord, cerebellum, or olfactory bulb, depending on whether they are relevant to the location of the device implantation. Use a brain block (Ted Pella) and razor blades to section the brain and create a flat plane closely matching the angle of the implanted devices; this is accomplished by placing the brain in an appropriately-sized brain block, orienting the brain such that any visible portion of the implant is parallel with the razor blade guide, and placing a razor blade in a blade guide at least $2 \mathrm{~mm}$ from the implant. Press the razor blade through the tissue. Mount this surface to a vibratome platform. Alternatively, a razor blade mounted to a micromanipulator can be used in a similarly controlled fashion as the brain block to create a plane closely matching the direction of the implant.

7. Place ice under the vibratome platform, and, after allowing the tissue surface to briefly dry on a paper towel, adhere the brain to a vibratome using Super Glue. Glue the flat tissue plane created in the previous step is to the stage. With asymmetric tissue dimensions, orient the sample such that the widest dimension is glued down parallel with the movement direction of the vibratome blade to help avoid the blade potentially knocking the tissue over. After the glue sets $(\sim 1-2 \mathrm{~min})$, add chilled $\left(\sim 4{ }^{\circ} \mathrm{C}\right)$ PBS to the vibratome dish around the tissue.

8. Collect thick tissue slices between $200-400 \mu \mathrm{m}$, including control tissue, using the maximum vibration rate $(\sim 100 \mathrm{~Hz})$ and a slow blade progression rate $\left(<0.2 \mathrm{~mm}\right.$ per second), with a blade angle of $10^{\circ}$ from horizontal. Collect slices carefully with a brush or scoop spatula and store in HBHS in a 24 well plate at $4{ }^{\circ} \mathrm{C}$.

9. Once the in situ device is visible to the unaided eye or a surgical microscope, collect thinner sections to approach the device in slice increments of $100 \mu \mathrm{m}$ or less.

Note: Typical silicon micro-implants are visible with the unaided eye in formaldehyde-fixed rodent cerebral cortex tissue between 300 and 500 $\mu \mathrm{m}$ from the device surface.

10. With the in situ device now close to the surface of the tissue, collect a $>250 \mu \mathrm{m}$ tissue slice containing the device within. Estimation of the necessary thickness can be aided by referencing previously collected slices.

Note: Larger devices, multishank devices, and angled devices may require thicker tissue slices ( $>500 \mu m$ ) to capture the device in one tissue piece; remember that both the penetration of applied labels and the microscopy imaging depth will be limited in the eventual data collection from these very thick slices. If possible, avoid striking the device with the vibratome blade, as this can cause dragging of the device through the tissue and morphological deformation.

\section{Tissue Processing and Clearing}

1. Wash slices $3 x$ at 5 min per wash in HBHS

2. Incubate in sodium borohydride ( $5 \mathrm{mg} \mathrm{NaBH}_{4} / 1 \mathrm{ml}$ of HBHS) for $30 \mathrm{~min}$ total (15 min each side of the slice). Flip the slices using a stainless steel or Teflon coated micro spoon and small paintbrush (Ted Pella). Slow and thoughtful movements improve the success of each slice flip. Avoid touching any areas around the implanted device with the brush. When possible, adding significantly more solution than required ( $>2 \mathrm{ml}$ ) allows one to manipulate and flip the tissue slices more easily.

Note: This step reduces endogenous autofluorescence; skip this step if fluorescent labels were applied during perfusion or xFP transgenic markers are present.

3. Wash slices $3 x$ at 5 min per wash in Wash Solution at room temperature. 
Note: Agitation during wash and incubation steps is optional. The authors speculate that subtle jarring of the stiff implant with respect to the surrounding brain tissue may occur with agitation. However, an orbital mixer moving at a gentle rate $(<60 \mathrm{rpm})$ may avoid this while increasing the movement of solutions.

4. Block for $2 \mathrm{hr}$ in Wash Solution at room temperature (flip slices after one hour).

5. Incubate in primary antibodies (diluted in Wash Solution) for approximately $48 \mathrm{hr}\left(24 \mathrm{hr}\right.$ on each side of the slice) at $4{ }^{\circ} \mathrm{C}$.

6. Perform 6 quick 3 min washes with Wash Solution.

7. Perform $6,1 \mathrm{hr}$ washes ( 3 washes on each side of the tissue slice) in Wash Solution (store at $4{ }^{\circ} \mathrm{C}$ if washing overnight).

8. Incubate in secondary antibodies (diluted with Wash Solution) for approximately $48 \mathrm{hr}\left(24 \mathrm{hr}\right.$ on each side) in $4{ }^{\circ} \mathrm{C}$.

9. Perform 6 quick 3 min washes with Wash Solution.

10. Perform 6, $1 \mathrm{hr}$ washes ( 3 washes on each side) in Wash Solution; store at $4{ }^{\circ} \mathrm{C}$ if washing overnight.

11. Remove Wash Solution and add the glycerol-based "U2 - Scale" clearing solution. Allow to clear approximately 1 week for thick slices $(250-500 \mu \mathrm{m})$ or $2-3$ weeks for very thick tissue sections $(>500 \mu \mathrm{m})$ at $4{ }^{\circ} \mathrm{C}$.

12. Cover plate with foil and store at $4{ }^{\circ} \mathrm{C}$.

13. Mount in slides accommodating thick tissue sections (Figure $\mathbf{2 b}, \mathbf{2 c}$ ) using clearing solution as mounting media and sealing with clear nail polish. Store at $4{ }^{\circ} \mathrm{C}$ away from light.

\section{Panorama Imaging of Full Tissue and Device Interface}

1. Using longer-working distance microscope objectives $(>2 \mathrm{~mm})$, begin imaging with a laser scanning confocal microscope or 2-photon microscope. We will demonstrate with a Zeiss LSM 710, Carl Zeiss "Zen" software (2010), and a computer-controlled XY translating stage to image a 3D panorama around an implant.

Note: Correct for the refractive index of the clearing solution either in software, through a corrective collar on the objective, or in data processing after collection. In this demonstration we enter a refractive index value for the "U2" clearing solution of 1.4 into the Leica Zen microscope software; this setting subtly adjusts z-step intervals to account for refractive index mismatch.

2. In tissue near the device, roughly assess the appropriate $z$-axis window and data collection settings in the $z$-dimension for each fluorescent channel using low laser power ( $\sim 0.5$ to $5 \%)$ and large z-step sizes $(>25 \mu \mathrm{m})$.

Note: Include additional space above and below when setting the z-axis when setting up for panoramic data collection, as the tissue may not lie entirely flat on the slide glass ( $20 \mu \mathrm{m}$ each direction).

3. Set the objective at the $x-y$ center of your eventual panorama; using the Zen panorama software, determine the number of collection positions required for each axis ( $x$ and $y$ ) to cover your region of interest.

4. Reassess and finalize the z-axis collection window.

5. Manually set the detector sensitivity and laser power to ramp appropriately with increased depth; the aim is typically to maintain approximately the same image intensity regardless of imaging depth into the tissue slice, but to also avoid high background noise.

6. Collect each fluorescent image data series. If necessary to avoid overlapping signals, run laser lines sequentially.

Note: If available, set the software to automatically save data to the hard drive during collection.

7. Change the software settings to collect the "reflectance" and "transmittance" data, and use a longer, more penetrative wavelength laser (e.g $633 \mathrm{~nm}$ ) to capture these channels. Determine the appropriate laser power and detector sensitivity for "reflectance" and "transmittance" collection and repeat the same collection series around the implanted device.

8. Export the data for later processing, quantification, and analysis.

\section{Representative Results}

Brain-implanted MEAs can be collected in tissue slices by first separating any skull-mounted components from the brain-embedded components.

Figure 2a shows the results of removing one side of a dental cement headcap and a portion of Kwik-Sil surrounding a MEA cable on a rat skull.

A soldering iron was used to remove the dental cement and Kwik-Sil in a fume hood. The cabling and any non-implanted MEA structures were next cut with micro-scissors by slowly excavating through the Kwik-Sil down to the surface of the fixed brain.

After slicing, labeling, and clearing tissue, simple custom-made slides are useful for mounting the thick tissue slices (Figure 2b). A mounted brain slice containing an implanted microdevice is shown in Figure 2c. Imaging through either side of the slide can allow you to assess the interface around a device, as demonstrated in Figure 3, where microglia along the silicon backing of an MEA are visible from one side (Figure 3a) and microglia along electrode sites and traces are visible on the other side (Figure $3 \mathbf{b}$ ).

Once mounted, tissue can be imaged using an XY translation stage. Overviews of fluorescent labels across entire tissue slices can be generated at the desired resolution (Figure 4). Detailed examination of the morphologically preserved tissue interface around implanted microdevices can be collected under high magnification for analysis of the local tissue response (Figure 5). 


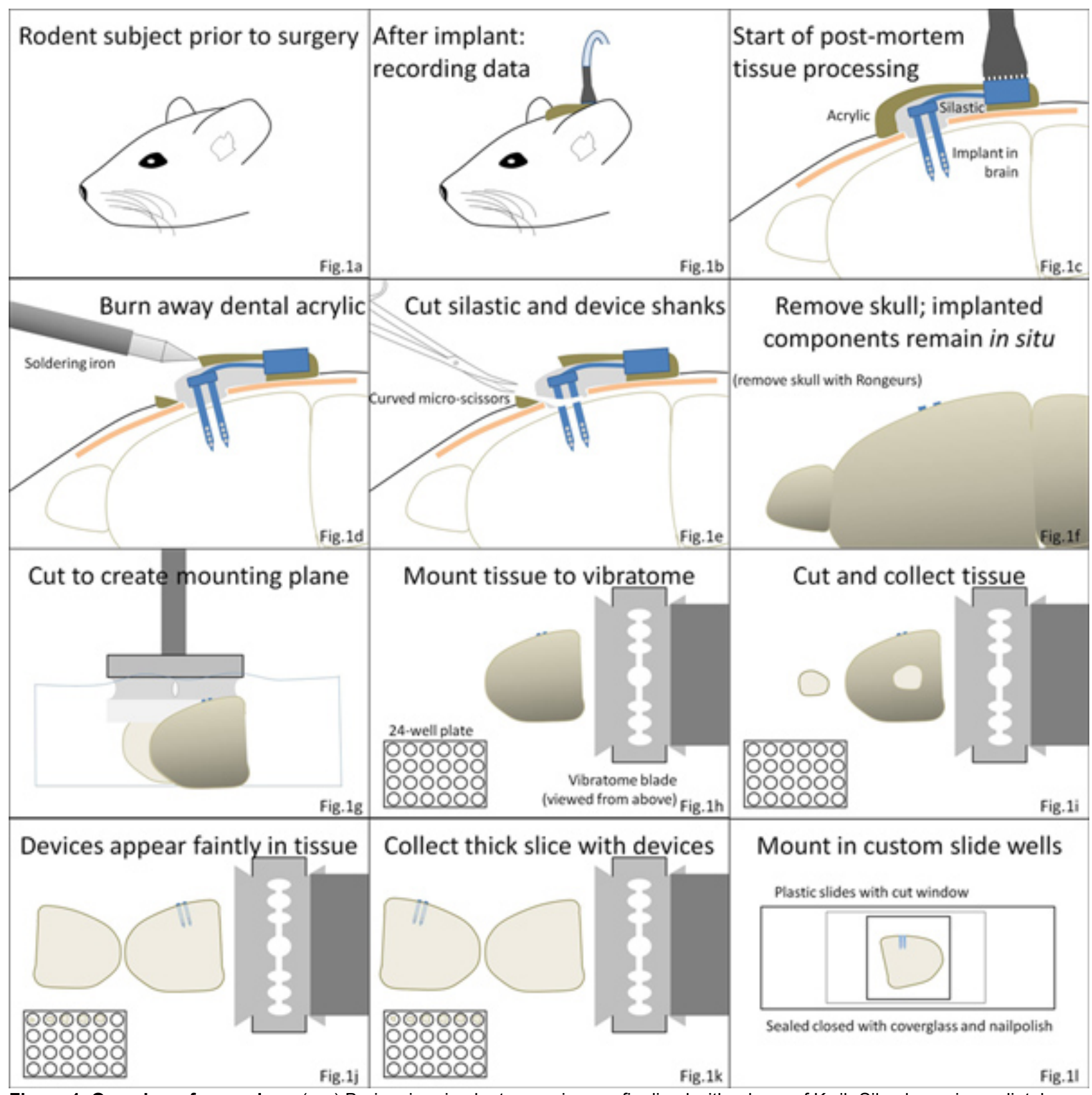

Figure 1. Overview of procedure. (a-c) Brain micro-implant surgeries are finalized with a layer of Kwik-Sil polymer immediately surrounding the device, followed by an acrylic cement covering. (d, e) Following euthanasia, the acrylic layer is burned away, and skull-mounted components of the device are separated from implanted components by cutting through the silicone elastomer. (f, $\mathbf{g})$ The brain is then removed from the skull and is cut and mounted to the vibratome stage. (h-k) Tissue slices are collected, including a tissue slice containing the device. (I) Tissue can then be processed and mounted in custom chambers for detailed microscopy. Click here to view larger figure. 

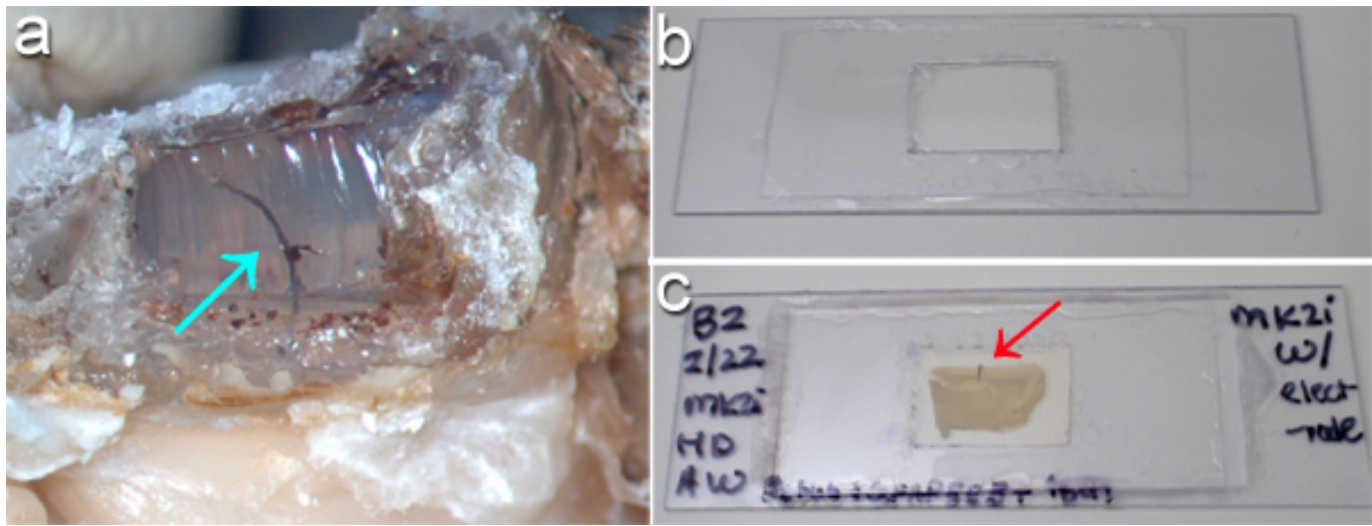

Figure 2. (a) Clear Kwik-Sil silicone elastomer surrounding an electrode-array cable (arrow) has been exposed by burning away a window in the dental cement cap on a perfused rodent head using a soldering iron. (b) To accommodate imaging into either side of a thick tissue slice, simple "slide-chambers" can be made by cutting a plastic slide and adhering coverglass to either side around the tissue and mounting solution. (c) A rat brain tissue slice with intact implant (red arrow) is shown after mounting. Either side of the tissue is quickly accessible to microscopy imaging with this setup. For scale, panel (a) is $25 \mathrm{~mm}$ across at the bottom, while panels (b, $\mathbf{c})$ are $80 \mathrm{~mm}$ across at the bottom.

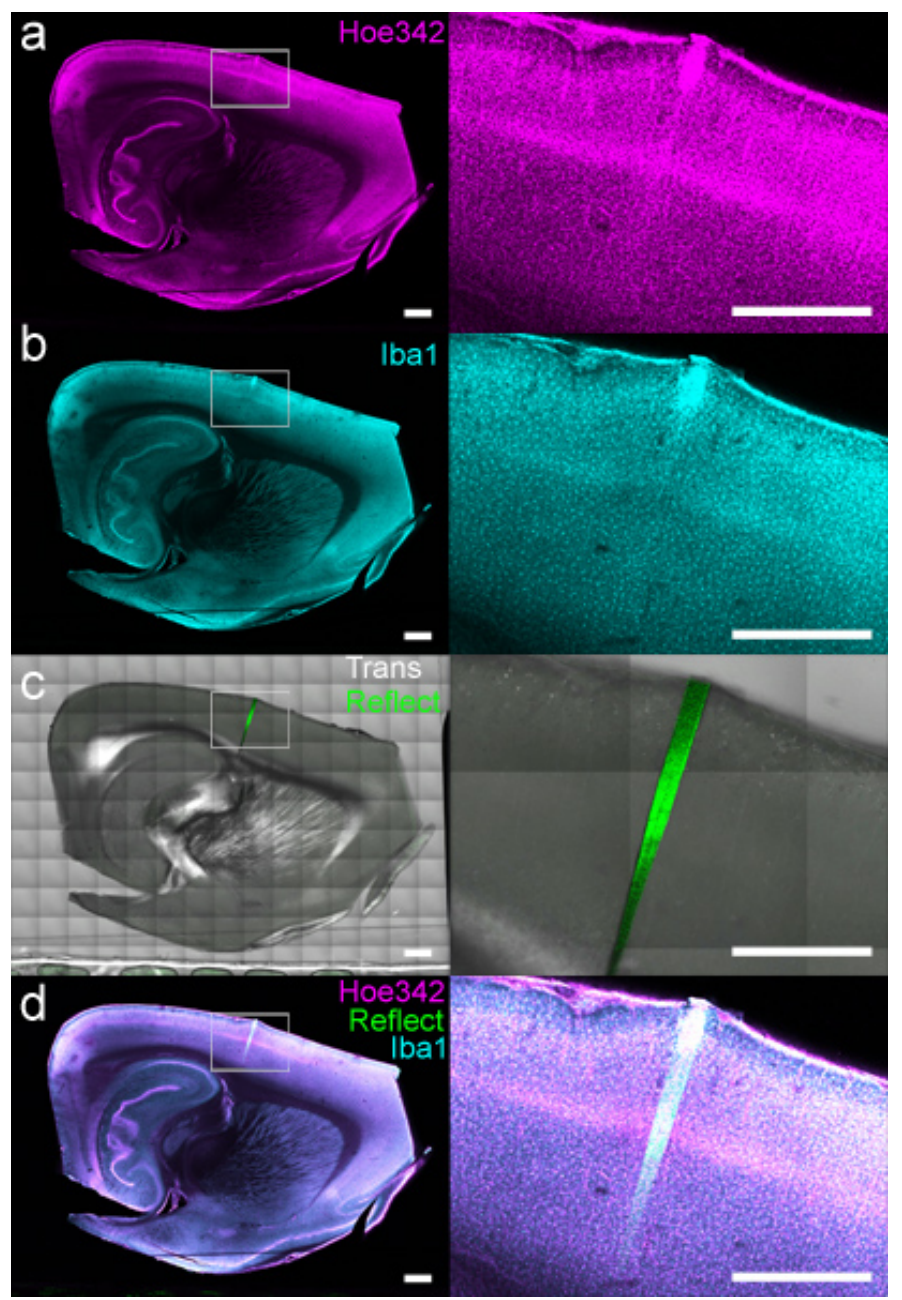

Figure 3. Maximum intensity z-projections of $40 \mu \mathrm{m}$ thick image stacks, collected around the backside (a) and front (b) of an implanted microelectrode array. Microglia (labeled with anti-lba1 and Alexa Fluor 633, white) and laser light reflectance (red), collected from the surface of the device, were sequentially imaged from both sides of a $400 \mu \mathrm{m}$ thick tissue slice. As the brain implants are often opaque, mounting with optical access to both sides provides a clear view of labeled tissue structures "behind" the implant with respect to the microscope objective. Scale bar $50 \mu \mathrm{m}$. 


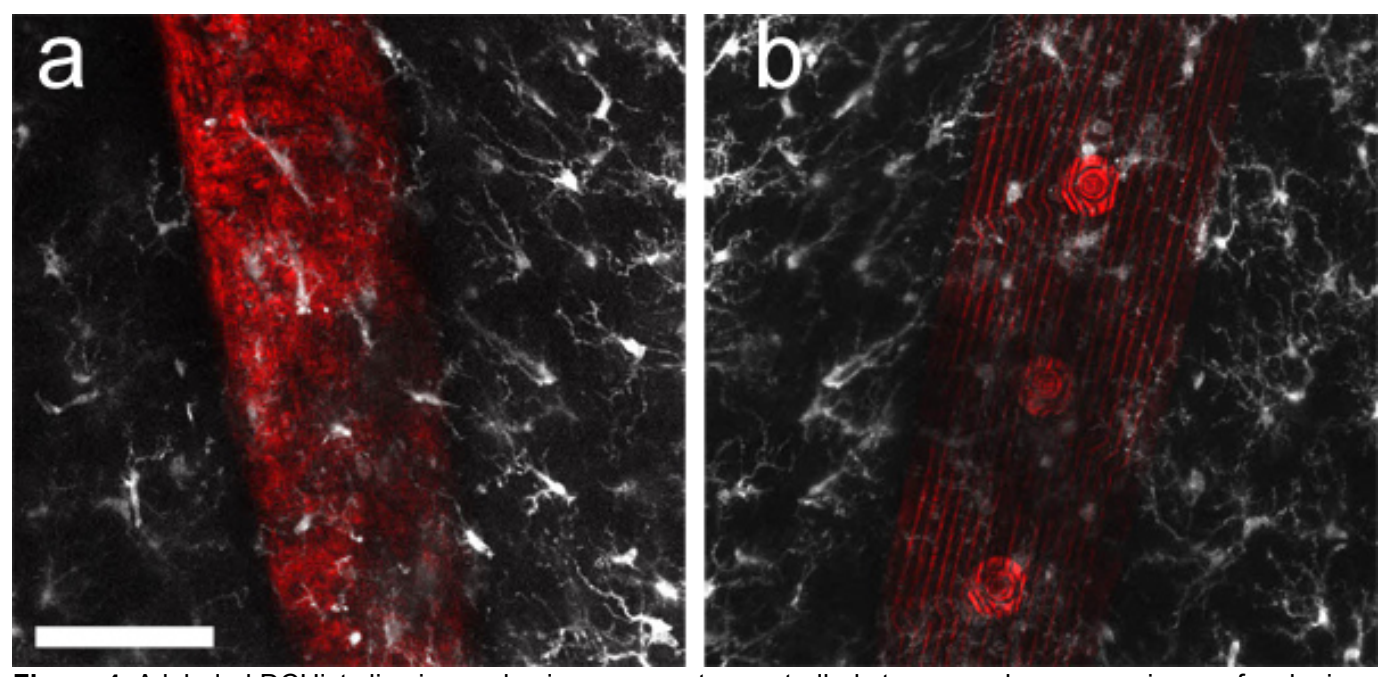

Figure 4. A labeled DCHist slice imaged using a computer-controlled stage on a laser scanning confocal microscope. (a) Cell nuclei (stained with Hoechst 33342) and (b) monocytes/microglia (labeled with anti-lba1) were simultaneously imaged on separate channels. (c) Transmission light and reflectance were also collected, showing the location of the 4-week implant with respect to Hoechst and Iba1 data. (d) Overlay of all channels but transmission is also shown. The white rectangle area is expanded in the images appearing at right. Scale bar $1 \mathrm{~mm}$.
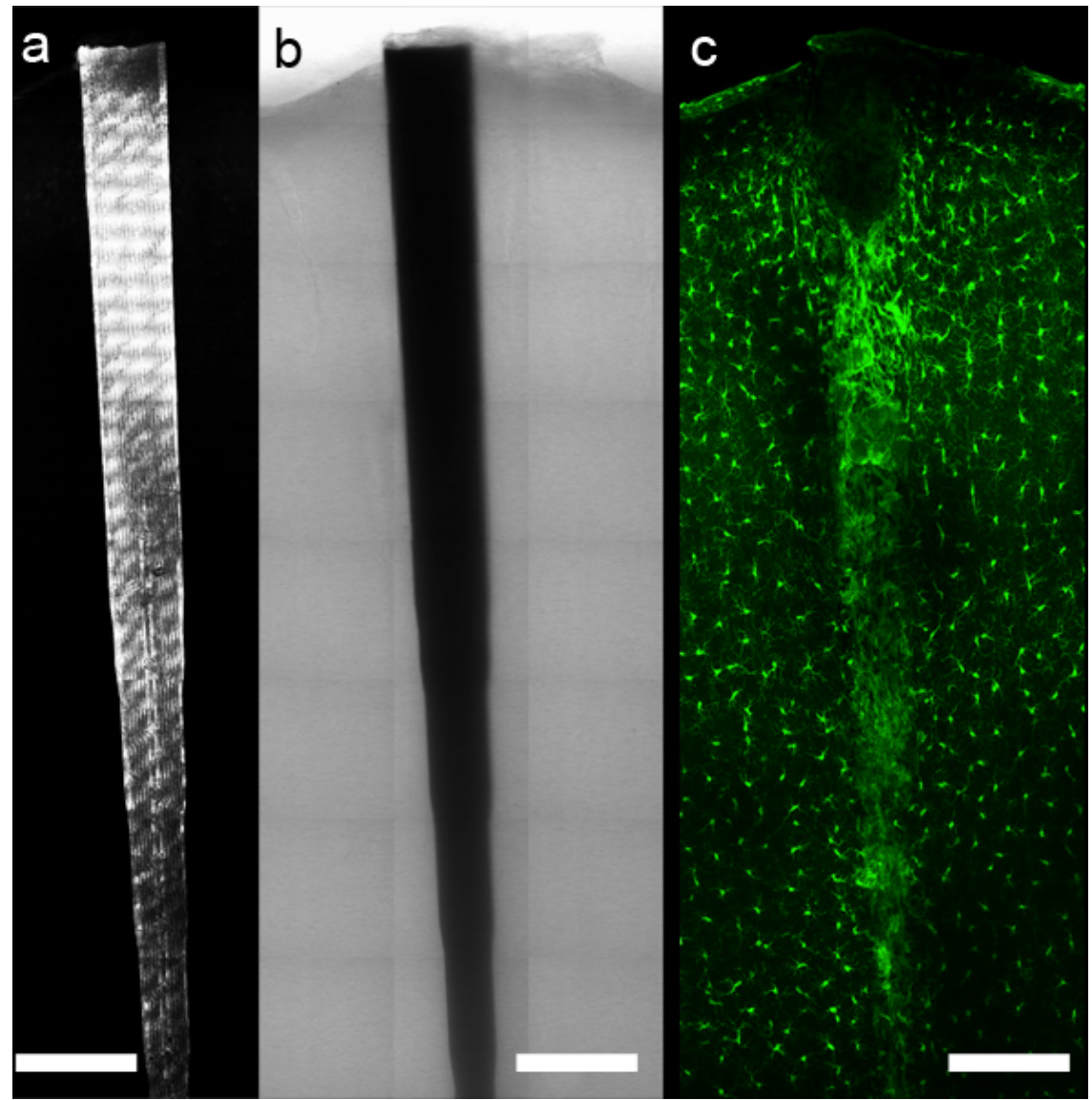

Figure 5. Using a computer-controlled microscope stage and appropriate software, panoramic imaging data can be collected around the implant Reflectance (a) and transmittance (b) allow localization of the device, while fluorescent antibody or chemical labels (c, anti-lba1 labeling of microglia and macrophages) allow detailed imaging of tissue components along the intact tissue interface. Scale bar $200 \mu \mathrm{m}$.

\section{Discussion}

The "Device-Capture Histology" (DCHist) method demonstrated here enables the close histological assessment of morphologically preserved interactions between brain tissue and tissue implants. DCHist tissue collection requires careful separation of skull-mounted device components from components implanted in the brain. DCHist also requires collection of a thick histological tissue slice (>250 $\mu \mathrm{m})$. These tissue sections, once labeled, cleared, mounted, and imaged, can provide novel insights into the implantation injury or subsequent chronic response to indwelling 
devices. Using advanced microscopy tools, the interface between tissue and device can be imaged and analyzed in high detail as shown in Figures 3-5.

The presented techniques in their current form rely on the ability to slowly excavate away the headcap made from two-part dental cement and Kwik-Sil down to the point of device implantation. Utilizing dental cement that can be burned away or otherwise removed and a clear silicon elastomer through which small scissors can be guided visually greatly aid in successfully separating the implanted device from its skull-mounted components. Attempting to cut the device under the skull and above the brain in order to collect it in situ is not recommended, as the skullmounted implant will be pulled out of the brain a significant amount.

Although thinner, smaller implants, such as single shank MEAs from NeuroNexus Technologies, are most amenable to DCHist, the principles are not restricted to single device shanks or microelectrode arrays. Collection and imaging strategies are broadly applicable to multi-shank devices and larger implants such as cannulas, with the requirements being that they must be separated from any skull mount and collected within a thick tissue section. Although the authors are focused on analysis of tissue surrounding cortical implants, devices driven deeper into the brain could also be collected and imaged. The depth of implant insertion should not affect the capture of the implant in a slice provided the device does not deviate wildly from the known angle of insertion.

Limitations exist to the useful application of the DCHist method. Brain tissue sections are challenging to image through hundreds of micrometers, especially in areas of white matter, although optical clearing solutions can greatly improve imaging depths in various tissues ${ }^{21}$. To further improve imaging depth, two-photon excitation microscopy may be employed along with the optical clearing described.

Another potential limitation of the described method can be the specific fluorescent immunohistochemistry methods and antibodies employed by researchers. Passive diffusion typically drives the incorporation of these markers through fixed tissue and onto antigen binding sites. Final antibody working concentrations must be determined by researchers on a case by case basis to maximize label penetration while avoiding high levels of background labeling. Antibody labeling should not be variable between slices that are processed identically, but different antibodies may vary considerably in their ability to penetrate and tag their antigen, with some antibodies easily labeling antigens many hundreds of micrometers deep and others labeling antigens only tens of micrometers deep. We describe improving this diffusion by applying antibody labels at higher than typical concentrations, for multiple days of application, and in a solution containing dilute detergent and blocking serum. Periodically flipping the slices also promotes even labeling. Antigen retrieval steps and alternative fixation processes (e.g. glutaraldehyde, microwave, etc.) may be appropriate for specific antigens. Secondary antibody-fluorochrome conjugates may also vary in their performance labeling thick sections, though this has not been observed by the authors using Alexa Fluor labels from Invitrogen. Alternatively, transgenic animal subjects expressing fluorescent proteins in the cell types of interest may be utilized to avoid issues with antibody label penetration, as many fluorescent proteins, such as eGFP, retain their fluorescence after formaldehyde treatment, and may be immediately visualized in tissue sections.

DCHist is a powerful set of techniques to capture and analyze the impact of implanted microdevices on brain tissue. Coupling this histological protocol with in vivo assessment of electrophysiology quality and electrode impedance data ${ }^{22}$ could greatly improve our understanding of the biological sources of physiology variability and degradation. The field of implanted neural prosthetic devices in particular may benefit from the detailed DCHist imaging of the intact device/tissue interface to inform further development of biologically neutral MEA devices.

\section{Disclosures}

The authors have no relevant disclosures to declare, financial or otherwise.

\section{Acknowledgements}

All experiments were done under the supervision of the Purdue Animal Care and Use Committee and the Laboratory Animal Program at Purdue University.

This work was sponsored by the Defense Advanced Research Projects Agency (DARPA) Microsystems Technology Office (MTO), under the auspices of Dr. Jack W. Judy (jack.judy@darpa.mil) as part of the Reliable Neural Technology Program, through the Space and Naval Warfare Systems Command (SPAWAR) Systems Center (SSC) Pacific Grant No. N66001-11-1-4013.

The authors would like to thank Mikhail Slipchenko, Don Ready, Greg Richter, Aaron Taylor, and Kevin Eliceiri for sharing their microscopy expertise.

\section{References}

1. Schwartz, A.B. Cortical Neural Prosthetics. Annual Review of Neuroscience. 27, 487-507 (2004).

2. Normann, R.A. Technology Insight: future neuroprosthetic therapies for disorders of the nervous system. Nature Clinical Practice Neurology. 3, 444-452 (2007).

3. Rousche, P. \& Normann, R.A. Chronic recording capability of the Utah Intracortical Electrode Array in cat sensory cortex. Journal of Neuroscience Methods. 82, 1-15 (1998).

4. Koivuniemi, A., Wilks, S.J., Woolley, A.J., \& Otto, K.J. Multimodal, longitudinal assessment of intracortical microstimulation. Prog. Brain Res. 194, 131-144 (2011).

5. Otto, K.J., Rousche, P.J., \& Kipke, D.R. Microstimulation in auditory cortex provides a substrate for detailed behaviors. Hearing Research. 210, 112-117 (2005).

6. Drake, K., Wise, K., Farraye, J., Anderson, D., \& BeMent, S. Performance of planar multisite microprobes in recordingextracellular single-unit intracortical activity. IEEE Transactions on Biomedical Engineering. 35, 719-732 (1988). 
7. Liu, X., et al. Stability of the interface between neural tissue and chronically implanted intracortical microelectrodes. IEEE Trans. Rehab. Eng. 7, 315-326 (1999).

8. Williams, J.C., Hippensteel, J.A., Dilgen, J., Shain, W., \& Kipke, D.R. Complex impedance spectroscopy for monitoring tissue responses to inserted neural implants. J. Neural Eng. 4, 410-423 (2007).

9. Polikov, V., Tresco, P., \& Reichert, W. Response of brain tissue to chronically implanted neural electrodes. Journal of Neuroscience Methods. 148, 1-18 (2005).

10. Szarowski, D.H., et al. Brain responses to micro-machined silicon devices. Brain Research. 983, 23-35 (2003).

11. McConnell, G.C., et al. Implanted neural electrodes cause chronic, local inflammation that is correlated with local neurodegeneration. $J$. Neural Eng. 6, 056003 (2009).

12. Holecko, M.M., Williams, J.C., \& Massia, S.P. Visualization of the intact interface between neural tissue and implanted microelectrode arrays. J. Neural Eng. 2, 97-102 (2005).

13. Pierce, A.L., Sommakia, S., Rickus, J.L., \& Otto, K.J. Thin-film silica sol-gel coatings for neural microelectrodes. J. Neurosci. Methods. 180, 106-110 (2009).

14. Wilks, S. Poly(3,4-ethylene dioxythiophene) (PEDOT) as a micro-neural interface material for electrostimulation. Frontiers in Neuroengineering. 2, (2009).

15. Johnson, M.D., Otto, K.J., \& Kipke, D.R. Repeated voltage biasing improves unit recordings by reducing resistive tissue impedances. IEEE Trans Neural Syst. Rehabil. Eng. 13, 160-165 (2005).

16. Otto, K.J., Johnson, M.D., \& Kipke, D.R. Voltage pulses change neural interface properties and improve unit recordings with chronically implanted microelectrodes. IEEE Trans. Biomed. Eng. 53, 333-340 (2006).

17. Hama, H., et al. Scale: a chemical approach for fluorescence imaging and reconstruction of transparent mouse brain. Nature Neuroscience. 14, 1481-1488 (2011).

18. Woolley, A.J., Desai, H.A., Steckbeck, M.A., Patel, N.K., \& Otto, K.J. In situ characterization of the brain-microdevice interface using Device Capture Histology. Journal of Neuroscience Methods. 201, 67-77 (2011).

19. Gage, G.J., Kipke, D.R., \& Shain, W. Whole Animal Perfusion Fixation for Rodents. J. Vis. Exp. (65), e3564, doi:10.3791/3564 (2012).

20. Li, Y., et al. Direct labeling and visualization of blood vessels with lipophilic carbocyanine dye Dil. Nature Protocols. 3, 1703-1708 (2008).

21. Clendenon, S.G., Young, P.A., Ferkowicz, M., Phillips, C., \& Dunn, K.W. Deep Tissue Fluorescent Imaging in Scattering Specimens Using Confocal Microscopy. Microscopy and Microanalysis. 17, 614-617 (2011).

22. Wilks, S.J., Richner, T.J., Brodnick, S.K., Kipke, D.R., Williams, J.C., \& Otto, K.J. Voltage Biasing, Cyclic Voltammetry, \& Electrical Impedance Spectroscopy for Neural Interfaces. J. Vis. Exp. (60), e3566, doi:10.3791/3566 (2012). 